\title{
Phase Structure and Critical Behavior of Multi-Higgs U(1) Lattice Gauge Theory in Three Dimensions
}

\author{
Tomoyoshi Ono ${ }^{1}$, Shunsuke Doi ${ }^{2}$, Yuki Hori ${ }^{2}$, Ikuo Ichinose ${ }^{1}$ and \\ Tetsuo Matsui ${ }^{2}$ \\ ${ }^{1}$ Department of Applied Physics, Graduate School of Engineering, \\ Nagoya Institute of Technology, Nagoya, 466-8555 Japan \\ ${ }^{2}$ Department of Physics, Kinki University, Higashi-Osaka, 577-8502 Japan \\ abstract
}

We study the three-dimensional (3D) compact $\mathrm{U}(1)$ lattice gauge theory coupled with $N$-flavor Higgs fields by means of the Monte Carlo simulations. This model is relevant to multi-component superconductors, antiferromagnetic spin systems in easy plane, inflational cosmology, etc. It is known that there is no phase transition in the $N=1$ model. For $N=2$, we found that the system has a second-order phase transition line $\tilde{c}_{1}\left(c_{2}\right)$ in the $c_{2}$ (gauge coupling) $-c_{1}$ (Higgs coupling) plane, which separates the confinement phase and the Higgs phase. Numerical results suggest that the phase transition belongs to the universality class of the 3D XY model as the previous works by Babaev et al. and Smiseth et al. suggested. For $N=3$, we found that there exists a critical line similar to that in the $N=2$ model, but the critical line is separated into two parts; one for $c_{2}<c_{2 \mathrm{tc}}=2.4 \pm 0.1$ with first-order transitions, and the other for $c_{2 \text { tc }}<c_{2}$ with second-order transitions, indicating the existence of a tricritical point. We verified that similar phase diagram appears for the $N=4$ and $N=5$ systems. We also studied the case of anistropic Higgs coupling in the $N=3$ model and found that there appear two second-order phase transitions or a single second-order transition and a crossover depending on the values of the anisotropic Higgs couplings. This result indicates that an "enhancement" of phase transition occurs when multiple phase transitions coincide at a certain point in the parameter space. 


\section{Introduction}

There are many interesting physical systems involving multi-component ( $N$-component) matter fields. Sometimes they are associated with exact or approximate symmetries like "flavor" symmetry. In some cases, the large- $N$ analysis or the $1 / N$ expansion[1] for an $N$-flavor system is applicable and it gives us useful information that cannot be obtained by the ordinary perturbative calculations. But the properties of the large- $N$ systems may differ from those at medium values of $N$ that one actually wants to know. Study of the $N$-dependence of various systems is certainly interesting, but has not been examined well.

Among these "flavor" physics, the effect of matter fields upon gauge dynamics is of quite general interest in quantum chromodynamics, strongly correlated electron systems, quantum spins, etc. $[2$, $3,4,5]$. In the present paper, we shall study the three-dimensional (3D) $\mathrm{U}(1)$ gauge theory with multi-component Higgs fields $\phi_{a}(x) \equiv\left|\phi_{a}(x)\right| \exp \left(i \varphi_{a}(x)\right)(a=1, \cdots, N)$ with fixed amplitudes $\left|\phi_{a}(x)\right|=1$. This model is of general interest, and knowledge of its phase structure, order of its phase transitions, etc. may be useful to get better understanding of various physical systems. These systems include the following:

$N$-component superconductor: Babaev[6] argued that under a high pressure and at low temperatures hydrogen gas may become a liquid and exhibits a transition from a superfluid to a superconductor. There are two order parameters; $\phi_{e}$ for electron pairs and $\phi_{p}$ for proton pairs. They may be treated as two complex Higgs fields $(N=2)$. In the superconducting phase, both $\phi_{e}$ and $\phi_{p}$ develop an off-diagonal long-range order, while in the superfluid phase, only the neutral order survives; $\lim _{|x| \rightarrow \infty}\left\langle\phi_{e}(x) \phi_{p}(0)\right\rangle \neq 0$.

p-wave superconductivity of cold Fermi gas: Each fermion pair in a $p$-wave superconductor has angular momentum $J=1$ and the order parameter has three components, $J_{z}=-1,0,1$. They are regarded as three Higgs fields $(N=3)$. As the strength of attractive force between fermions is increased, a crossover from a superconductor of the BCS type to the type of Bose-Einstein condensation is expected to take place[7].

Phase transition of $2 D$ antiferromagnetic(AF) spin models: In the $s=1 / 2 \mathrm{AF}$ spin models, a phase transition occurs from the Néel state to the valence-bond solid state as parameters are varied. Senthil et al.[8] argued that the effective theory describing this transition take a form of $\mathrm{U}(1)$ gauge theory of spinon $\left(C P^{1}\right)$ field $z_{a}(x)\left(\left|z_{1}\right|^{2}+\left|z_{2}\right|^{2}=1\right)$ with an additional Berry phase and a "deconfined phase" of spinons appears at the critical point. If one consider the easy-plane limit $\left(S_{z}=0\right),\left|z_{1}\right|^{2}=\left|z_{2}\right|^{2}=1 / 2$ and the $C P^{1}$ bosons are expressed by two Higgs fields as $z_{a}=\exp \left(i \varphi_{a}\right) / \sqrt{2}(N=2)$. This system is studied by Nogueira et al. by a renormalization group 
analysis[9] and it is clarified that the above easy-plane limit has only a first-order phase transition.

Similar limit to the above may be taken to relate the superconductivity of ultracold fermionic atoms with spin $J$ to the $\mathrm{U}(1)$ gauge model with $N$ Higgs fields. J. Zhao, et al. considered the $S U(N)$ Hubbard model to describe the superconductivity of fermionic atoms, which has a $N=$ $2 J+1$-component order parameter[10]. At large repulsion $U$ and at the filling factor $n=1 / N$, the model becomes the $\mathrm{U}(1)$ gauge model with $C P^{N-1}$ spins. A $C P^{N-1}$ variable is parametrized as $z_{a}=\rho_{a} \exp \left(i \varphi_{a}\right)$ with $\sum_{a=1}^{N} \rho_{a}^{2}=1$. In the symmetric limit, which is the easy-plane limit for $N=2$, $\rho_{a}^{2}=1 / N$ and $z_{a}$ becomes a Higgs field.

Effects of doped fermionic holes (holons) to these AF spins were also studied extensively. The effective theory obtained by integrating out holon variables may be a $\mathrm{U}(1)$ gauge theory with $N=2$ Higgs fields (with nonlocal gauge interactions). Kaul et al.[11] predicts that such a system exhibits a second-order transition, while numerical simulations of Kuklov et al.[12] exhibit a weak first-order transition. This point should be clarified in future study.

Inflational cosmology: In the inflational cosmology[13], a set of Higgs fields is introduced to describe a phase transition and inflation in early universe. Plural Higgs fields are necessary in a realistic model[14].

In the rest of the present paper, we shall study the multi-Higgs lattice models by Monte Carlo(MC) simulations. We consider the simplest form of the model, i.e., the 3D compact lattice gauge theory without Berry's phase. We introduce the Higgs fields $\phi_{x a}$ on the site $x$ of the cubic lattice and treat them in the London limit, $\left|\phi_{x a}\right|=1, \phi_{x a}=\exp \left(i \varphi_{x a}\right)$. We also put the compact $\mathrm{U}(1)$ gauge field $U_{x \mu}=\exp \left(i \theta_{x \mu}\right)$ on the link $(x, x+\mu) . \mu(=1,2,3)$ is the direction index (we use them also as the unit vectors). The action $S$ consists of the Higgs coupling with its coefficients $c_{1 a}(a=1, \ldots, N)$ and the plaquette term with its coefficient $c_{2}$ as

$$
S=\frac{1}{2} \sum_{x, \mu} \sum_{a=1}^{N}\left(c_{1 a} \phi_{x+\mu, a}^{\dagger} U_{x \mu} \phi_{x a}+\text { H.c. }\right)+\frac{c_{2}}{2} \sum_{x, \mu<\nu}\left(U_{x \nu}^{\dagger} U_{x+\nu, \mu}^{\dagger} U_{x+\mu, \nu} U_{x \mu}+\text { H.c }\right) \text {. }
$$

The partition function $Z$ of the model is given by

$$
\begin{aligned}
Z & =\int[d U][d \phi] \exp (S), \\
\int[d U][d \phi] & =\prod_{x, \mu} \int_{-\pi}^{\pi} \frac{d \theta_{x \mu}}{2 \pi} \prod_{x, a} \int_{-\pi}^{\pi} \frac{d \varphi_{x a}}{2 \pi} .
\end{aligned}
$$

A couple of models close to Eqs.(1.1) and (1.2) have been investigated. Smiseth et al.[15] studied the noncompact $\mathrm{U}(1)$ Higgs models. A duality transformation maps the charged sector into the inverted $X Y$ spin model. Thus they predicted that the system exhibits a single inverted $X Y$ transition and $N-1 X Y$ transitions. Their numerical study confirmed this prediction for $N=2$. For 
$N=2$, Kragset et al.[16] studied the effect of Berry's phase term in the compact Higgs model. They reported that Berry's phase term suppresses monopoles (instantons) and changes the second-order phase transitions to first-order ones.

The phase structure of the present system (1.1) can be studied by the following consideration developed by Smiseth et al.[15, 17]. That is, among $N$ phases $\varphi_{a}(x)$ of the Higgs fields, the sum $\tilde{\varphi}_{+} \equiv \sum_{a} \varphi_{a}$ couples to the gauge field and describes charged excitations, whereas the remaining $N-1$ independent linear combinations $\tilde{\varphi}_{i}(i=1, \cdots, N-1)$ describe neutral excitations. The latter $N-1$ modes may be regarded as a set of $N-1 X Y$ spin models. As the $N=1$ compact $\mathrm{U}(1)$ Higgs model stays always in the confinement phase[18], we expect $N-1$ second-order transitions of the type of the $X Y$ model. The above discussion is useful to get an intuitive picture of the phase structure. In the present paper, we shall study the system (1.1) by means of MC simulation and verify the above conclusion.

The rest of the present paper is organized as follows. In Sec.2, we shall study the $N=2$ multiHiggs model and report the results of the MC simulations, in particular, its phase diagram in the $c_{1}-c_{2}$ plane. We measure the internal energy, specific heat and instanton density. In Sec.3, we shall investigate the $N=3$ cases. By varying the ratios of the three Higgs couplings, the model exhibits interesting phase structure. In Sec.4, we present the result of the cases of $N=4$ and $N=5$. Section 5 is devoted for conclusion.

\section{Two-flavor Higgs model $(N=2)$}

For the MC simulations, we used the standard Metropolis algorithm[19]. We consider the cubic lattice with the periodic boundary condition and its size $L^{3}$ up to $L=36$. The typical statistics used was $6 \cdot 10^{5} \mathrm{MC}$ steps and the averages and errors were estimated over 20 samples.

We first study the $N=2$ case with symmetric couplings $c_{11}=c_{12} \equiv c_{1}$. We measured the internal energy $U \equiv-\langle S\rangle / L^{3}$ and the specific heat $C \equiv\left\langle(S-\langle S\rangle)^{2}\right\rangle / L^{3}$ in order to obtain the phase diagram and determine the order of phase transitions.

In Fig.1(a), we show $C$ at $c_{2}=0.4$ as a function of $c_{1}$ for $L=22,28,32$. The peak of $C$ develops as the system size is increased. The results indicate that a second-order phase transition occurs at $c_{1} \simeq 0.91$. In fact, we applied the finite-size-scaling (FSS) hypothesis to $C$ of Fig.1(a) in the form of

$$
C\left(c_{1}, L\right)=L^{\sigma / \nu} \eta\left(L^{1 / \nu} \epsilon\right)
$$

where $\epsilon=\left(c_{1}-c_{1 \infty}\right) / c_{1 \infty}$ and $c_{1 \infty}$ is the critical coupling at $L \rightarrow \infty$. We determined $\nu=$ $0.65 \pm 0.02, \sigma=0.16 \pm 0.02$, and $c_{1 \infty}=0.909 \pm 0.010$ with the scaling function $\eta(x)$ plotted in Fig.1(b). This result supports the FSS (2.1). 

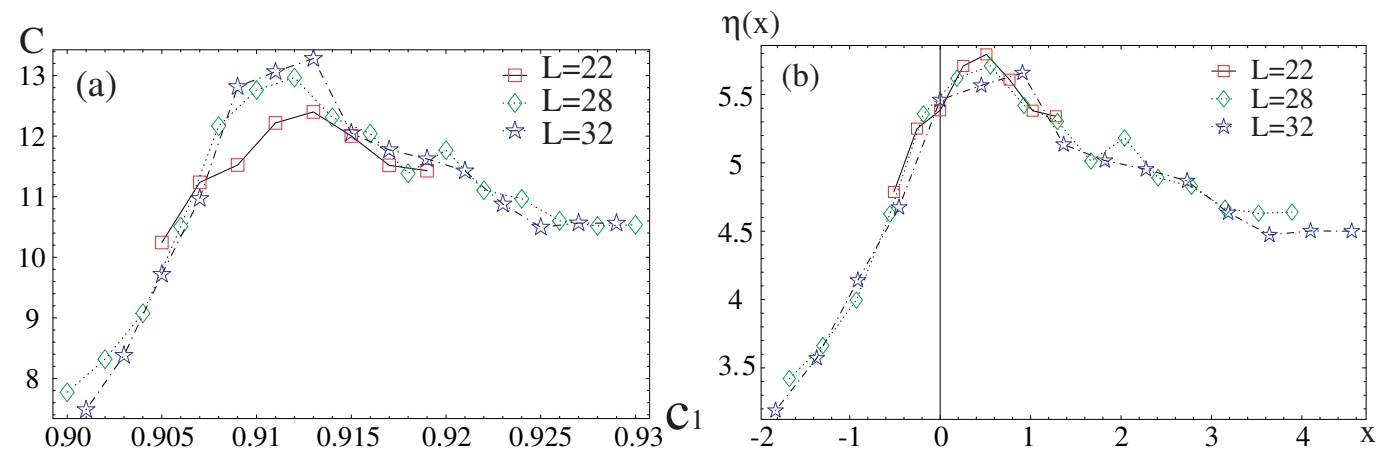

Figure 1: (a) System-size dependence of specific heat $C$ of $L=22,28,32$ for $N=2$ along $c_{2}=0.4$. (b) Scaling function $\eta(x)$ of Eq.(2.1) for Fig.1(a).

The above results for $N=2$ are consistent with the prediction given in the introduction. The sum $\tilde{\varphi}_{x+} \equiv \varphi_{x 1}+\varphi_{x 2}$ couples with the compact gauge field and generates no phase transition[18], while the difference $\tilde{\varphi}_{x-} \equiv \varphi_{x 1}-\varphi_{x 2}$ behaves like the angle variable in the 3D $X Y$ model. The 3D $X Y$ model has a second-order phase transition with the critical exponent $\nu=0.666 \ldots[20]$. Our value of $\nu$ obtained above is consistent with this value. In fact, the same result was previously obtained in Ref.[16], which studied the $N=2$ model on the specific line $c_{1}=c_{2}$ in the $c_{2}-c_{1}$ plane by means of the MC simulations of large system sizes ${ }^{1}$.

It is instructive to see the behavior of the instanton density $\rho$ in order to study the gauge dynamics at the phase transition point. We employ the definition of $\rho$ in the 3D $\mathrm{U}(1)$ compact lattice gauge theory given by DeGrand and Toussaint[22]. $\rho$ in Fig.2 decreases very rapidly near the phase transition point at $c_{1} \simeq 0.91$. This indicates that a "crossover" from dense to dilute instanton "phases" is accompanied with the phase transition. In other words, the observed phase transition can be interpreted as a confinement(small $\left.c_{1}\right)$-Higgs(large $c_{1}$ ) phase transition.

The above conclusion is supported by the following consideration. An effective gauge model $S_{\text {eff }}(U)$ is obtained by integrating out the Higgs fields $\phi_{x a}$ in $Z$ of Eq.(1.2),

$$
Z=\int[d U] \exp \left[S_{\mathrm{eff}}(U)\right]
$$

\footnotetext{
${ }^{1}$ The $N=2$ model with a noncompact gauge action was studied by Motrunich et al.[21]. Phase stucture of that mode was also clarified by the paper by Kragset et al. [16].
} 


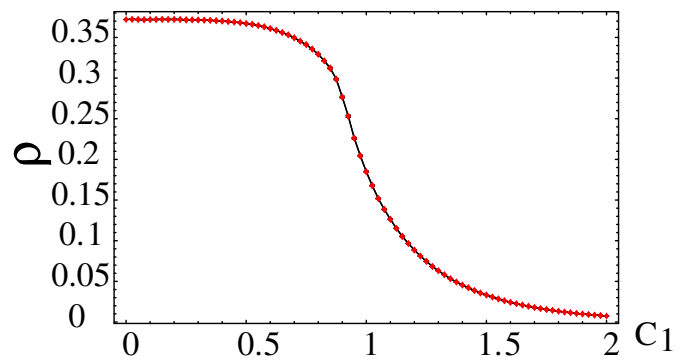

Figure 2: Instanton density $\rho$ for $N=2$ at $c_{2}=0.4$ as a function of $c_{1}$. System size $L=16$.

$$
\exp \left[S_{\text {eff }}(U)\right] \equiv \int[d \phi] \exp [S(U, \phi)]
$$

For small $c_{1}$, the above integration over the Higgs fields can be performed by using the hopping expansion in powers of $c_{1}$. The resultant effective action $S_{\text {eff }}(U)$ contains nonlocal interaction terms of the gauge field $U_{x \mu}$. Recently we studied models of $\mathrm{U}(1)$ gauge field in 3D, which contain nonlocal interactions [3]. We found that the nonlocal terms give dominant effect on the gauge dynamics and a confinement-deconfinement phase transition takes place as their coefficients are getting large.

Knowledge of the phase structure of the $C P^{N-1}$ model is also useful to identify the phases in the present model. As explained in the introduction, the present model is the easy-plane limit of the $C P^{1}$ model. The $C P^{N-1}$ model in $3 \mathrm{D}$ was studied both analytically by means of the $1 / N$ expansion [23] and numerically by defining the model on the lattice [24, 4]. These studies show that the spontaneous breaking of the internal $S U(N)$ symmetry accompanys the phase transition to the Higgs phase of the gauge dynamics. Similarly in the present model, the observed second-order phase transition corresponds to the transition from the global U(1) symmetric phase (confinement phase) to the phase of the the spontaneous breaking of the global U(1) symmetry (Higgs phase).

In Fig.3, we present the phase diagram for $N=2$ in the $c_{2}-c_{1}$ plane. There exists a second-order phase transition line separating the confinement and the Higgs phases. There also exists a crossover line similar to that in the $3 \mathrm{D} N=1 \mathrm{U}(1)$ Higgs model[18]. 


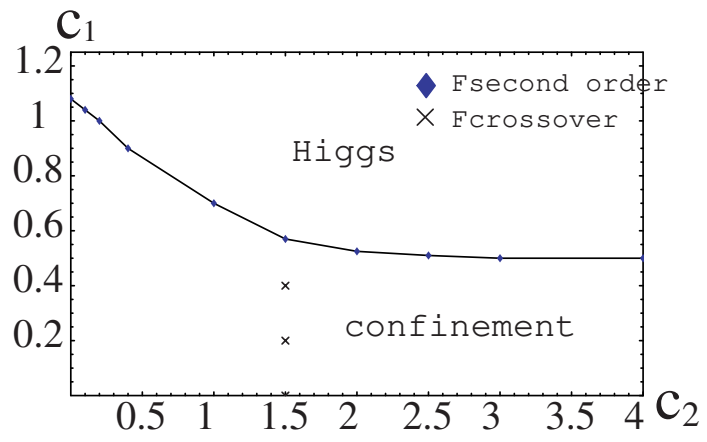

Figure 3: Phase diagram for $N=2$. There are two phases, confinement and Higgs, separated by a second-order phase transition line. There also exists a crossover line in the confinement phase separating dense and dilute instanton-density regions. Location of the phase transition line is determined from the data of $L=24$.

\section{Three-flavor Higgs model $(N=3)$}

\subsection{Symmetric case $c_{1 a}=c_{1}$}

Let us turn to the $N=3$ case. Among many possibilities of three $c_{1 a}$ 's, we first consider the symmetric case $c_{11}=c_{12}=c_{13} \equiv c_{1}$. One may expect that there are two $(N-1=2)$ second-order transitions that may coincide at a certain critical point. Studying the $N=3$ case is interesting from a general viewpoint of the critical phenomena, i.e., whether coincidence of multiple phase transitions changes the order of the transition. We studied various points in the $c_{2}-c_{1}$ plane and found that the order of transition changes as $c_{2}$ varies.

In Fig.4, we show $U$ and $\rho$ along $c_{2}=1.5$ as a function of $c_{1}$. Both quantities show hysteresis loops, which are signals of a first-order phase transition. In Fig.5, we present $C$ at $c_{2}=3.0$. The peak of $C$ at around $c_{1} \sim 0.48$ develops as $L$ is increased, whereas $U$ shows no discontinuity and hysteresis. Therefore, we conclude that the phase transition at $\left(c_{2}, c_{1}\right) \sim(3.0,0.48)$ is of second order.

In order to locate the tricritical point from the first to second phase transitions, we studied the region $c_{2}=2.0 \sim 2.5$ in detail. In Fig.6, we present the internal energy $U$ and the specific heat $C$ at $c_{2}=2.0,2.2,2.3,2.4,2.5$ for the system size $L=32$. $U$ at $c_{2}=2.0$ shows a hysteresis at $c_{1} \simeq 0.506 \sim 0.508$. As $c_{2}$ increases, the hysteresis becomes milder and at $c_{2}=2.5$ it almost disppears. Also, as $c_{2}$ increases, the region and height of the peak of $C$ measured from the smooth background become reduced. These behavior, together with the size dependence of $U$ and $C$, suggest 

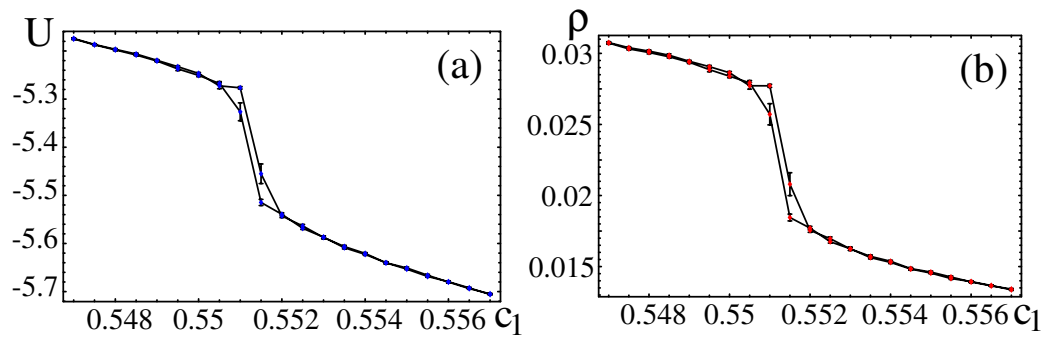

Figure 4: (a) Internal energy $U$ and (b) instanton density $\rho$ for $N=3$ at $c_{2}=1.5$ and $L=16$. Both exhibit hysteresis loops in the path where $c_{1}$ is first increased and then decreased by the step $\Delta c_{1}=0.0005$, indicating a first-order phase transition at $c_{1} \simeq 0.551$.
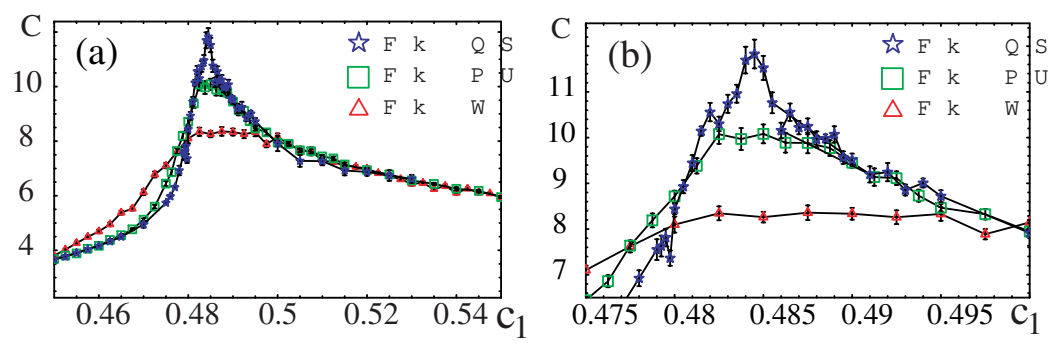

Figure 5: (a) Specific heat for $N=3$ at $c_{2}=3.0$. (b) Close-up view near the peak. The peak develops as $L$ increases.

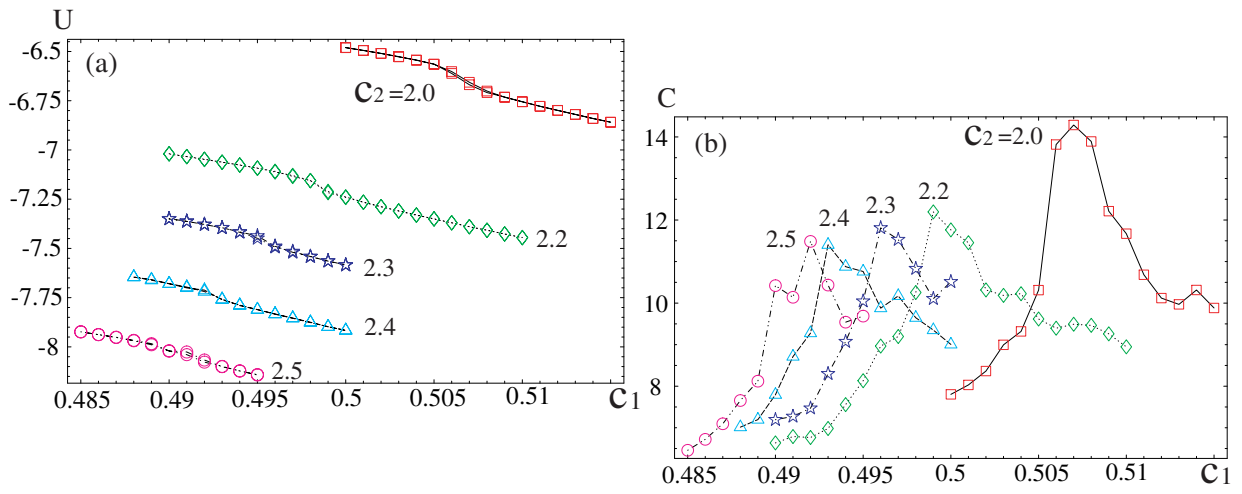

Figure 6: (a) Internal Energy $U$ and (b) Specific heat $C$ for $N=3$ and $L=32$ at $c_{2}=2.0 \sim 2.5$. (Values of $c_{2}$ are indicated near each curve.) As $c_{2}$ increases, the changes of $U$ become milder and the region and size of the peak of $C$ become reduced. 
that the change in the order of phase transition from the first-order one to the second-order one takes place in this region of $c_{2}$. To support this point, we also studied the distribution $\rho(E) \exp (-E)$ of the internal-energy, which is defined as

$$
Z=\int[d U][d \phi] \exp (S)=\int d E \int[d U][d \phi] \exp (S) \delta(S+E)=\int d E \rho(E) \exp (-E) .
$$

If the phase transition is first order, $\rho(E) \exp (-E)$ exhibits a double peak structure near the critical point, while a second-order transition exhibits a single peak structure. In Fig.7, we present the distribution of $\rho(E) \exp (-E)$ for $c_{2}=2.0 \sim 2.5$ and $L=32$.
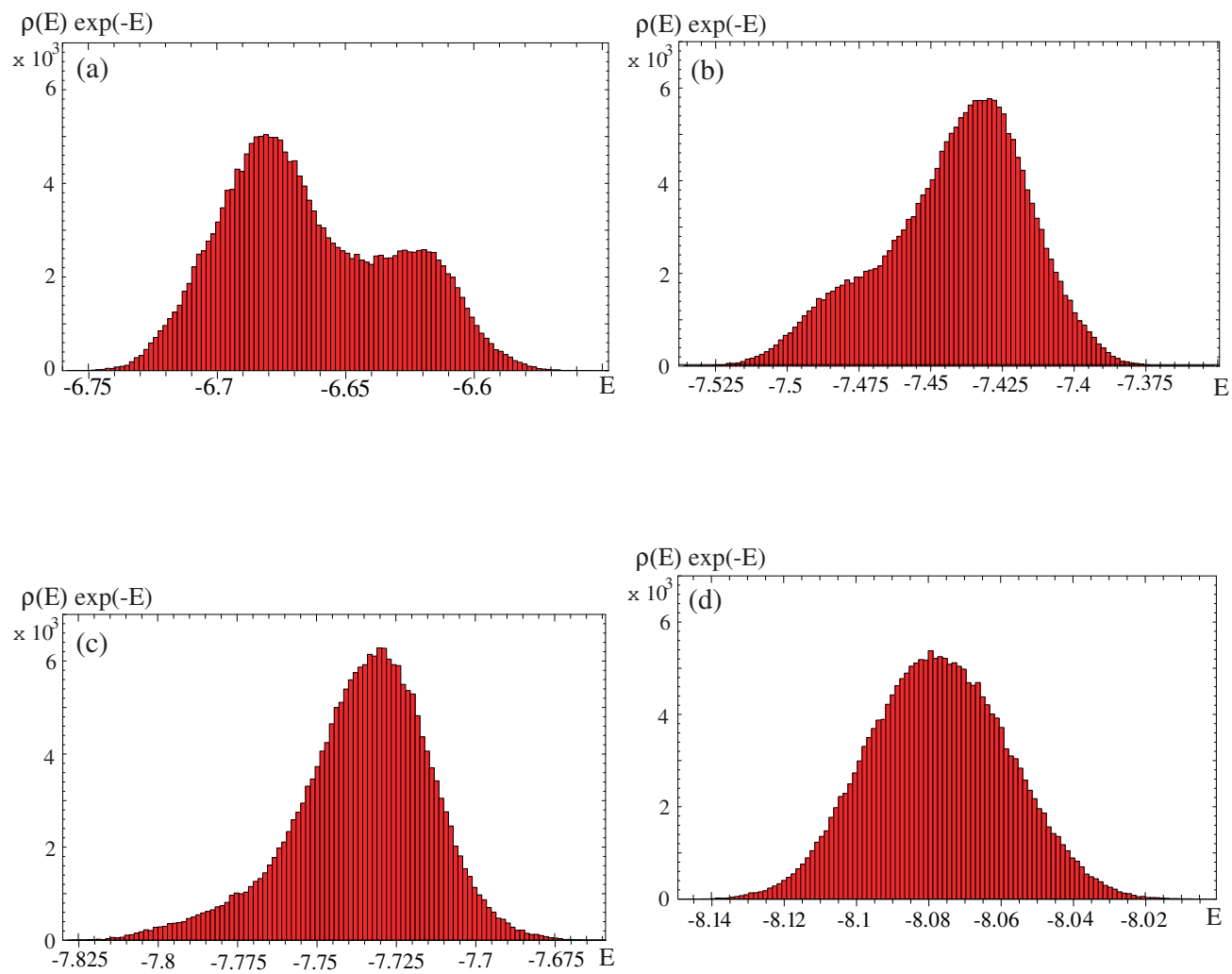

Figure 7: Distribution of the energy, $\rho(E) \exp (-E)$ of Eq.(3.1), for $N=3$ and $L=32$. (a) $\left(c_{2}, c_{1}\right)=(2.0,0.507)$; (b) $\left(c_{2}, c_{1}\right)=(2.3,0.495)$; (c) $\left(c_{2}, c_{1}\right)=(2.4,0.493)$; (d) $\left(c_{2}, c_{1}\right)=(2.5,0.492)$. The signal of double-peak structure, which is shown clearly in Fig.(a), becomes weaker as $c_{2}$ increases, and disappears in Fig.(d). 
The value of $c_{1}$ is chosen near the peak location of $C$ given in Fig.6(b). Apparently, Fig.7(a), the distribution at $c_{2}=2.0$, fits better by a double-peak (e.g., double Gaussian) distribution rather than by a single-peak one. The fact that two peaks here have different weights mainly reflects that it is slightly away from the critical point of $c_{1}$. Fig.7(c) for $c_{2}=2.4$ has a single peak at $E \simeq-7.73$, but not symmetric around this peak, which shows a remnant of the second peak at lower $U$ region. Fig.7(d) for $c_{2}=2.5$ shows a clean single-peak distribution. From these observations we determine that the tricritical point is located at $c_{2}=c_{2 \mathrm{tc}} \simeq 2.4 \pm 0.1$.

In Fig.8(a), we present the phase diagram of the symmetric case for $N=3$, where the order of transition between the confinement and Higgs phases changes from first $\left(c_{2}<c_{2 t c}\right)$ to second order $\left(c_{2 \mathrm{tc}}<c_{2}\right)$. In Fig.8(b) we present $C$ along $c_{1}=0.2$, which shows a smooth nondeveloping peak. As shown in Fig.9, the instanton density $\rho$ decreases smoothly around this peak. These results indicate a crossover at $c_{2} \simeq 1.5$.
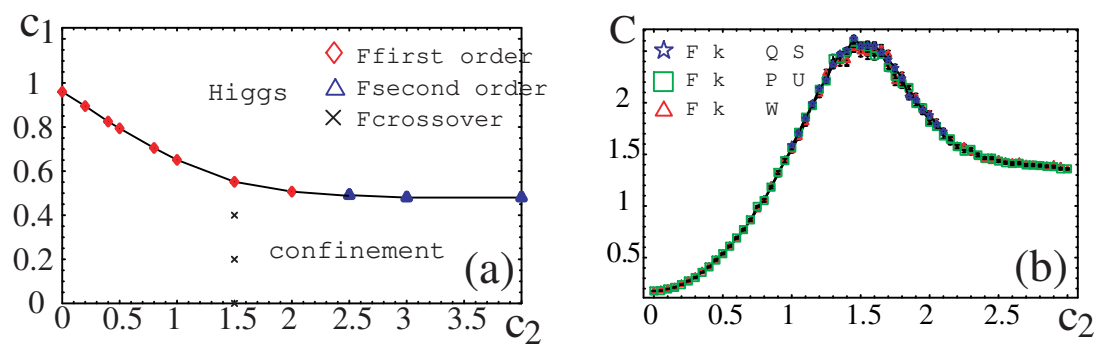

Figure 8: (a) Phase diagram for the $N=3$ symmetric case. The phase transitions are first order in the region $c_{2}<c_{2 \mathrm{tc}} \simeq 2.4$, whereas they are second order in the region $c_{2}>c_{2 \mathrm{tc}}$. There exists a tricritical point at around $\left(c_{2}, c_{1}\right) \sim(2.4,0.49)$. Crosses near $c_{2}=1.5$ line show crossovers. (b) Specific heat for $N=3$ at $c_{1}=0.2$. It has a system-size independent smooth peak at which a crossover takes place.

\section{$3.2 \quad$ Asymmetric case}

Then it becomes interesting to consider asymmetric cases, e.g., $c_{11} \neq c_{12}=c_{13}$. This case is closely related to a doped $\mathrm{AF}$ magnet. $\phi_{2}$ and $\phi_{3}$ correspond there to the $C P^{1}$ spinon field in the deep easyplane limit, whereas $\phi_{1}$ corresponds to doped holes (although they are fermionic). This case is also relevant to cosmology because the order of Higgs phase transition in the early universe is important in the inflational cosmology. Furthermore, one may naively expect that once a phase transition to 


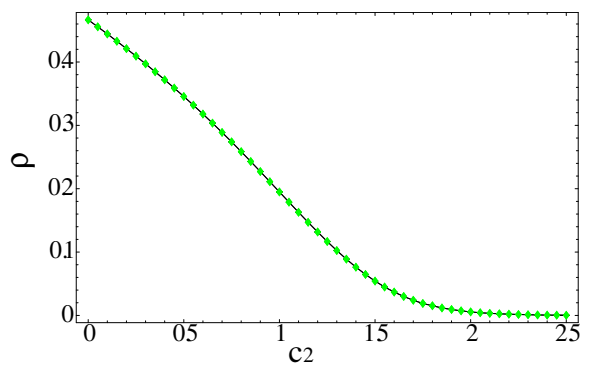

Figure 9: Instanton density $\rho$ in the $N=3$ symmetric case for $c_{1}=0.2$ as a function of $c_{2}$. System size $L=16$.

the Higgs phase occurs at certain temperature $T$, no further phase transitions take place at lower $T$ 's even if the gauge field couples with other Higgs bosons. However, our investigation below will show that this is not the case.

Below we shall consider the two cases, (i) $c_{12}=c_{13}>c_{11}$ and (ii) $c_{12}=c_{13}<c_{11}$. Let us first consider the case (i) $c_{12}=c_{13}=2 c_{11}$, which we call the $c_{1}=(1,2,2)$ model, and focus on the case $c_{2}=1.0$. As shown in Fig.10(a), $C$ exhibits two peaks at $c_{11} \sim 0.35$ and 0.52. Figs.10(b),(c) present the detailed behavior of $C$ near these peaks, which show that the both peaks develop as $L$ is increased. We conclude that both of these peaks show second-order transitions. This result is interpreted as the first-order phase transition in the symmetric $N=3$ model is decomposed into two second-order transitions in the $c_{1}=(1,2,2)$ model.

Let us turn to the opposite case (ii), $c_{12}=c_{13}=0.5 c_{11}$, i.e., the $c_{1}=(2,1,1)$ model at $c_{2}=1.0$. One may expect that two second-order phase transitions appear as in the previous $c_{1}=(1,2,2)$ model. However, the result shown in Fig.11 indicates that there exists only one second-order phase transition near $c_{11} \sim 1.08$. The broad and smooth peak near $c_{11} \sim 0.85$ shows no $L$ dependence and we conclude that it is a crossover. This crossover is similar to that in the ordinary $N=1$ gauge-Higgs system as we shall see by the measurement of $\rho$ below.

The orders of these transitions are understood as follows: In the $c_{1}=(1,2,2)$ model, as we increase $c_{11}$, the two modes $\phi_{x a}(a=2,3)$ with larger $c_{1 a}$ firstly become relevant and the model is effectively the symmetric $N=2$ model. The peak in Fig.10(b) is interpreted as that of the second-order phase transition in this model. As the Higgs couplings $c_{11}$ 's are increased further, the fluctuations of the gauge field is negligibly small, and the effective model is the $N=1 X Y$ model of $\phi_{x 1}$. It gives the second-order peak in Fig.10(c). Similarly, in the $c_{1}=(2,1,1)$ model, $\phi_{x 1}$ firstly becomes relevant. The effective model is the $N=1$ model, which gives the broad peak in the specific 

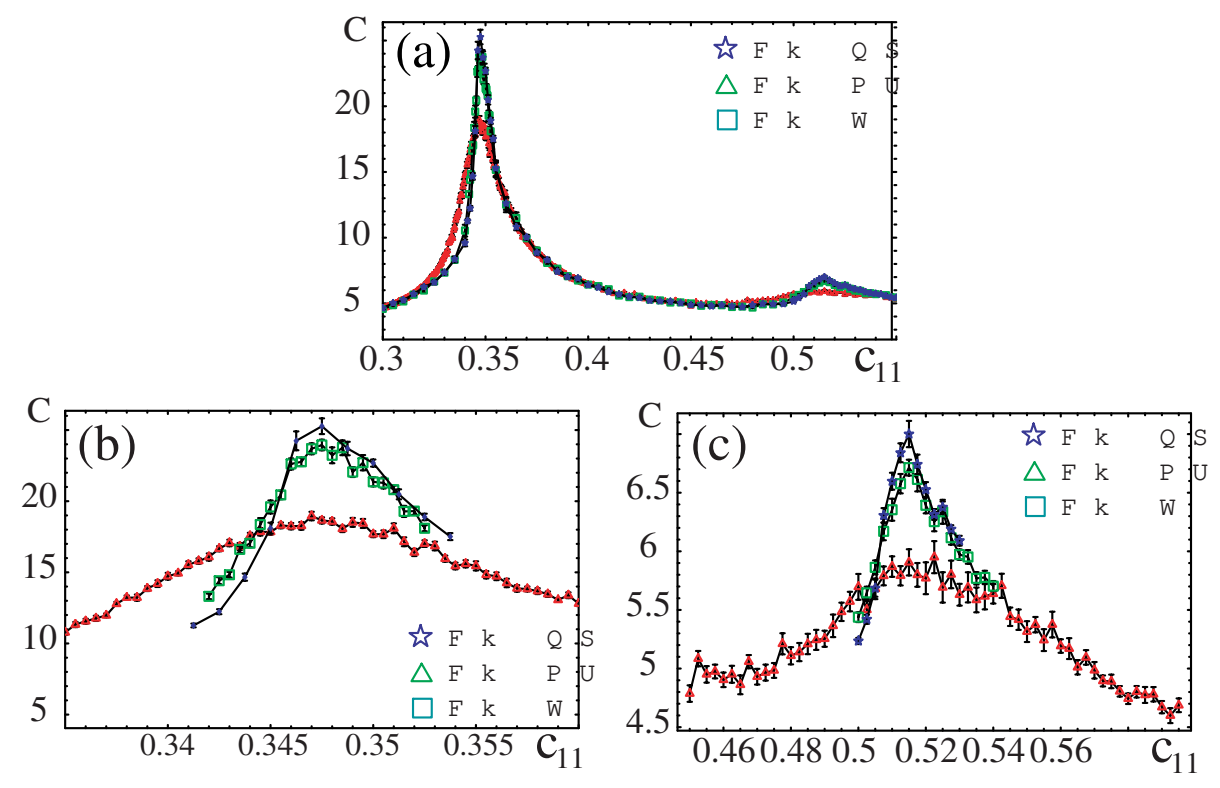

Figure 10: (a) Specific heat of the $c_{1}=(1,2,2)$ model $(N=3)$ at $c_{2}=1.0$. (b,c) Close-up views of $C$ near $(\mathrm{b}) c_{11} \sim 0.35$ and $(\mathrm{c}) c_{11} \sim 0.52$.

heat $C$ in Fig.11 as the crossover takes place there[18]. For larger values of $c_{11}$ 's, the effective model is the $N=2$ symmetric model of $\phi_{x 2}, \phi_{x 3}$ and $U_{x \mu}$, giving the sharp second-order peak in $C$ in Fig.11.

In Fig.12, we present $\rho$ of the $c_{1}=(1,2,2)$ and $(2,1,1)$ models as a function of $c_{11} . \rho$ of the $c_{1}=(1,2,2)$ model decreases very rapidly at around $c_{11} \sim 0.35$, which is the phase transition point in lower $c_{11}$ region. On the other hand, at the higher phase transition point, $c_{11} \sim 0.52, \rho$ shows no significant changes. This observation indicates that the lower- $c_{11}$ phase transition is the confinement-Higgs transition, whereas the higher- $c_{11}$ transition is a charge-neutral $X Y$-type phase transition.

On the other hand, $\rho$ of the $c_{1}=(2,1,1)$ model decreases rapidly at around $c_{11} \sim 0.85$, where $C$ exhibits a broad peak. This indicates that the crossover from the dense to dilute-instanton regions occurs there just like in the $N=1$ case[18]. No "anomalous" behavior of $\rho$ is observed at the critical point $c_{11} \sim 1.1$, and therefore the phase transition is that of the neutral mode. 


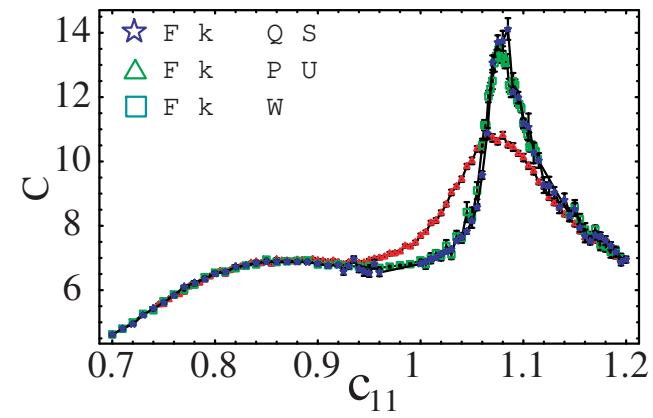

Figure 11: Specific heat of the $c_{1}=(2,1,1)$ model at $c_{2}=1.0$.

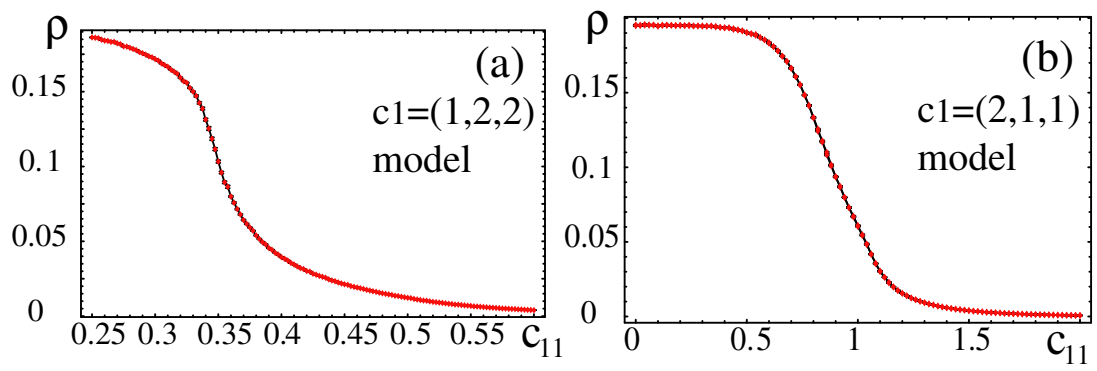

Figure 12: Instanton density $\rho$ at $c_{2}=1.0$ in the (a) $c_{1}=(1,2,2)$ model and (b) $c_{1}=(2,1,1)$ model. System size $L=16$.

\section{Symmetric model with $N=4$ and 5}

We have also studied the symmetric case for $N=4$ and 5 multi-Higgs models at $c_{2}=0$. Both cases show clear signals of first-order transitions at $c_{1} \simeq 0.89(N=4), 0.84(N=5)$ as shown in Fig.13. On the other hand, at $c_{2}=\infty$, the gauge dynamics is "frozen" to $U_{x \mu}=1$ up to gauge transformations, so there remain $N$-fold independent $X Y$ spin models, each of which exhibits a second-order transition at $c_{1} \simeq 0.46$. Thus we expect a tricritical point for general $N>2$ at some finite $c_{2}$ separating first-order and second-order transitions.

\section{Conclusion}

In the present paper, we studied the U(1) multi-flavor Higgs model in 3D, which is closely related to various interesting physical systems. By means of the MC simulations, we clarified its phase 

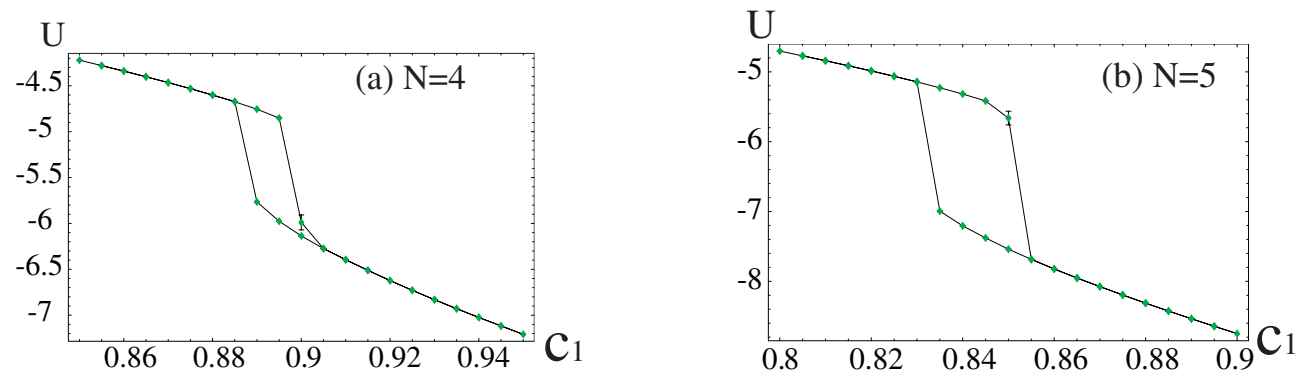

Figure 13: Internal energy $U$ in (a) the $N=4$ model and (b) $N=5$ model for $c_{2}=0$ with the system size $L=24$. Both exhibit hysteresis loops in the path where $c_{1}$ is first increased and then decreased by the step $\Delta c_{1}=0.005$. This indicates a first-order phase transition for $c_{2}=0$ at $c_{1} \simeq 0.89$ for $N=4$ and at $c_{1} \simeq 0.84$ for $N=5$.

structure and critical behavior. Let us summarize the results. For $N=2$ there is a critical line $\tilde{c}_{1}\left(c_{2}\right)$ of second-order transitions in the $c_{2}-c_{1}$ plane, which distinguishes the Higgs phase $\left(c_{1}>\tilde{c}_{1}\right)$ and the confinement phase $\left(c_{1}<\tilde{c}_{1}\right)$. This result is consistent with Kragset et al.[16]. We obtained the crtitical exponent of the phase transition by means of the FSS and found that the result is very close to that of the 3D XY model.

For $N=3$ there is a similar transition line, but the region $0<c_{2}<c_{2 \mathrm{tc}} \simeq 2.4$ is of first-order transitions while the region $c_{2 \mathrm{tc}}<c_{2}$ is of second-order transitions. We concluded that there exists a tricritical point. It is very interesting and also important to clarify the nature of the tricritical point, especially its critical exponent. This problem is under study and the result will be published in future.

To study the mechanism of generation of these first-order transitions, we studied the asymmetric cases and found two second-order transitions [in the $c_{1}=(1,2,2)$ model] or one crossover and one second-order phase transition [in the $c_{1}=(2,1,1)$ model]. The former case implies that two simultaneous second-order transitions strengthen the order to generate a first-order transition. Chernodub et al.[25] reported a similar generation of an enhanced first-order transition in a related 3D Higgs model with singly and doubly charged scalar fields. We stress that the above change of the order is dynamical because (1) It depends on the value of $c_{2}$, (2) Related 3D models, the $C P^{N-1}$ and $N$-flavor $C P^{1}$ gauge models, exhibit always second-order transitions (See Ref.[4]).

\section{Acknowledgements}


We thank K. Sakakibara and M.N.Chernodub for useful discussions. We also thank Y. Nakano for his assistance in numerical calculations. This work was partially supported by Grant-in-Aid for Scientific Research from Japan Society for the Promotion of Science under Grant No.20540264.

\section{References}

[1] See, e.g., S. Coleman, "Aspects of Symmetry" (Cambridge University Press 1985);

E. Brezin, S. R. Wadia, "The Large N Expansion in Quantum Field Theory and Statistical Physics" (World Scientific, Singapore 1993).

[2] Y. Iwasaki, K. Kanaya, S. Sakai, and T. Yoshie, Phys. Rev. Lett.69(1992)21.

[3] G. Arakawa, I. Ichinose, T. Matsui, K. Sakakibara, Phys. Rev. Lett. 94(2005)211601.

[4] S. Takashima, I. Ichinose, T. Matsui, Phys. Rev. B73(2006)075119.

[5] F.S. Nogueira and H.Kleinert, Phys.Rev. B77 (2008)045107, and references cited therein.

[6] E. Babaev, A. Sudbø, and N. W. Ashcroft, Nature 431(2004)666.

[7] Y. Ohashi, Phys. Rev. Lett. 94(2005)050403.

[8] T. Senthil, L. Balents, S. Sachdev, A. Vishwanath, and M. P. A. Fisher, Science 303(2004)1490; T. Senthil, A. Vishwanath, L. Balents, S. Sachdev, and M. P. A. Fisher, Phys. Rev. B70(2004)144407.

[9] F.S. Nogueira, S. Kragset, and A. Sudbø, Phys.Rev. B76(2007)220403(R).

[10] J. Zhao, K. Ueda, and X. Wang, Phys. Rev. B74(2006)233102.

[11] R. K. Kaul, A. Kolezhuk, M. Levin, S. Sachdev, and T. Senthil, arXiv:cond-mat/0611536.

[12] A. B. Kukulov, N. V. Prokof'ev, B. V. Svistunov, and M. Troyer, Ann. Phys. 321(2006)1602.

[13] A. H. Guth, Phys. Rev. D23(1981)347.

[14] R. Allahverdi, K. Enqvist, J. Carcia-Bellido, and A. Mazumdar, Phy.Rev.Lett. 97(2006)191304; J.C.B. Sanchez, K. Dimopoulos, D.H. Lyth, JCAP 0701(2007)015.

[15] J. Smiseth, E. Smørgrav, and A. Sudbø, Phys. Rev. Lett. 93(2004)077002;

See also E. Smørgrav, J. Smiseth, E.Babaev, and A. Sudbø, Phys. Rev. Lett. 94(2005)096401;

S. Sachdev, in "Quantum magnetism", U. Schollwock, J. Richter, D. J. J. Farnell and R. A. Bishop eds, Lecture Notes in Physics, Springer, Berlin (2004). 
[16] S. Kragset, E. Smørgrav, J. Hove, F. S. Nogueira, and A. Sudbø, Phys. Rev. Lett. 97(2006)247201.

[17] J. Smiseth, E. Smørgrav, E.Babaev, and A. Sudbø, Phys.Rev. B71(2005)214509; See also E.Babaev, L.P. Fadeev, and A.J. Niemi, Phys.Rev. B65(2002)100512(R).

[18] S. Wenzel, E. Bittner, W. Janke, A.M.J. Schakel, and A. Schiller, Phys. Rev. Lett. 95(2005)051601.

[19] N. Metropolis, A. W. Rosenbluth, M. N. Rosenbluth, A. M. Teller, E. Teller, J. Chem. Phys. $21(1953) 1087$.

[20] M. Campostrini, M. Hasenbusch, A. Pelissetto, P. Rossi, and E. Vicari, Phys. Rev. B63(2001)214503.

[21] O.I. Motrunich and A. Vishwanath, Phys.Rev. B70(2004)075104; See also Ref[17].

[22] T. A. DeGrand and D. Toussaint, Phys. Rev. D22(1980)2478.

[23] I.Ya. Areféva and S.I. Azakov, Nucl.Phys. 162(1980)298.

[24] S. Takashima, I. Ichinose, and T. Matsui, Phys.Rev. B72(2005)075112.

[25] M. N. Chernodub, E.-M. Ilgenfritz, and A.Schller, Phys. Rev. B73(2006)100506;

M. Bock, M. N. Chernodub, E.-M. Ilgenfritz, and A. Schller, Phys. Rev. B76(2007)184502. 